\title{
Involvement of Glutamine-238 in the Substrate Specificity of Human Laeverin/Aminopeptidase $\mathbf{Q}$
}

\author{
Yoshikuni Goto, ${ }^{a, b}$ Rina Yoshioka, ${ }^{a}$ Naomi Arisaka, ${ }^{a}$ Akira Hattori, ${ }^{c}$ and Masafumi Tsujimoto $* a, b$ \\ ${ }^{a}$ Laboratory of Cellular Biochemistry, RIKEN; 2-1 Hirosawa, Wako, Saitama 351-0198, Japan: baculty of \\ Pharmaceutical Sciences, Teikyo-Heisei University; 4-1 Uruido-minami, Ichihara, Chiba 290-0193, Japan: and \\ ${ }^{c}$ Department of System Chemotherapy and Molecular Sciences, Graduate School of Pharmaceutical Sciences, Kyoto \\ University; Kyoto 606-8501, Japan. \\ Received September 29, 2010; accepted October 18, 2010; published online October 20, 2010
}

\begin{abstract}
Human laeverin/aminopeptidase Q (APQ) is a novel member of the M1 family of zinc aminopeptidases and is specifically expressed on the cell surface of extravillous trophoblasts. In this study, we examined the significance of GIn-238 of laeverin/APQ, a putative S1 site residue, by site-directed mutagenesis for its enzymatic activity and substrate specificity. Replacement of Gln-238 with Ala caused a significant change in substrate specificity rather than a decrease in enzymatic activity. These results indicate that Gln-238 is important for the substrate specificity of laeverin/APQ. In addition, our data suggest that direct electrostatic interaction between substrate and $\mathrm{S} 1$ site of the enzyme is not involved in the mutant enzyme's preference for basic amino acids.
\end{abstract}

Key words aminopeptidase; laeverin; endoplasmic reticulum aminopeptidase 1; site-directed mutagenesis; substrate specificity

Aminopeptidases hydrolyze the N-terminal amino acid of proteins or peptide substrates. Among them, the M1 family of zinc aminopeptidases (gluzincins) shares the consensus GAMEN and $\operatorname{HEXXH}(X)_{18} E$ motifs essential for enzymatic activity. This family, which consists of 11 enzymes in humans, ${ }^{1-3)}$ plays important roles in several pathophysiological processes, such as angiogenesis, cell cycle regulation, reproduction, memory retention, blood pressure control, and antigen presentation to major histocompatibility complex (MHC) class I molecules. ${ }^{4-12)}$

Laeverin was originally identified as a cell-surface protein specifically expressed on human extravillous trophoblasts. ${ }^{13)}$ cDNA cloning of human laeverin revealed that it contains both consensus motifs and thus is a novel member of the M1 family. Another group also predicted the existence of the 'leaverin' gene in the human genome through a genomic search and named it aminopeptidase Q (APQ). ${ }^{14)}$ Recently, we established a large-scale production system of the enzyme and characterized its enzymatic properties in detail. ${ }^{15)} \mathrm{We}$ found that the enzyme degraded several placenta-derived peptide hormones, such as angiotensin III, endokinin C, and kisspeptin-10. Furthermore because its exopeptidase motif GAMEN is uniquely composed of the HAMEN sequence, we examined the enzymatic significance of His-379 of human laeverin/APQ by site-directed mutagenesis and found that this residue contributes to the unique properties of the enzyme. ${ }^{16)}$ Considering the susceptibility of these peptides and their specific expression in the placenta, we speculated that laeverin/APQ plays important roles in the maintenance of normal pregnancy in humans.

In our previous work, we identified a residue affecting enzymatic activity and the substrate specificity of endoplasmic reticulum aminopeptidase (ERAP)1, a member of the M1 aminopeptidase family. ${ }^{17)}$ In particular, replacement of Gln181 with Asp caused a marked change in the substrate specificity of ERAP1. On the other hand, replacement with Ala led to almost complete loss of enzymatic activity. To further analyze the residue affecting enzymatic activity and substrate specificity of the M1 family of aminopeptidases, we examined in the present study the effect of replacing Gln-238 of human laeverin/APQ with Ala. We found that Q238A laeverin/APQ retained substantial enzymatic activities with marked change of its substrate specificity, indicating the significance of this residue for the enzymatic characteristics of laeverin/APQ. Data presented in this study imply that direct electrostatic interaction is not involved in the mutant enzyme's preference for basic amino acids, which was suggested in the previous work. ${ }^{17)}$

\section{MATERIALS AND METHODS}

Molecular Modeling of Laeverin/APQ The published X-ray crystallographic structure of Thermoplasma acidophilum Tricorn-interacting factor F3 (TIFF3) (Protein Data Bank code $1 \mathrm{Z} 5 \mathrm{H})^{18)}$ was used as a template for modeling the catalytic site of ERAP1 with the three-dimensional Jigsaw Protein Server (http://www.bmm.icnet.uk/ 3djigsaw/). The structure was displayed using the CueMol program (Ishitani, R., CueMol: Molecular Visualization Framework, http://cuemol.sourceforge.jp).

Site-Directed Mutagenesis To obtain a large amount of recombinant human laeverin/APQ, we slightly modified its cDNA by polymerase chain reaction (PCR). Briefly, to express the enzyme as a soluble protein, the coding sequences for the cytosolic and transmembrane region of the enzyme $\left(\mathrm{Met}^{1}-\mathrm{Gln}^{64}\right)$ were replaced with that for human trypsin II signal peptide (i.e. MNLLLILTFVAAVAA). ${ }^{15)}$ Hexahistidine tag was added at its C-terminal end. Amplified cDNA was cloned into the BssHII-XhoI site of the baculovirus transfer vector pFastbac-1 (Invitrogen, CA, U.S.A.). The cDNA encoding mutant Q238A laeverin/APQ was generated using a QuickChange site-directed mutagenesis kit (Stratagene, La Jolla, CA, U.S.A.). The primers used to replace Gln-238 with Ala in human laeverin/APQ cDNA were as follows: sense primer, 5'-GCCCTGTTAGCGTCCGCTCTGGAACCAACATTTG-3', and antisense primer 5'-CAAATGTTGGTTCCAGAGCGGACGCTAACAGGGC-3'.

The sequences of the products were confirmed by automated sequencing on an Applied Biosystems model 3730.

Expression and Purification of Recombinant Wild- 
Type and Mutant Laeverin/APQs in the Baculovirus System The pFastbac-1 vectors containing laeverin/APQ cDNAs were transformed to competent DH10bac EScherichia coli cells harboring the baculovirus genome (bacmid) and a transposition helper vector (Invitrogen). Subsequently, insect Sf 9 cells were transfected with recombinant bacmids using the Cellfectin reagent (Invitrogen). After a 3-d incubation period, recombinant baculoviruses were isolated and used to infect Sf 9 cells at a multiplicity of infection of 0.1 . Three days after infection, the amplified viruses were harvested.

For the production of laeverin/APQ, Sf 9 cells were grown at $27^{\circ} \mathrm{C}$ in $100 \mathrm{ml} \mathrm{Sf-900III} \mathrm{medium} \mathrm{(Gibco,} \mathrm{CA,} \mathrm{U.S.A.)}$ and $1.5 \times 10^{6}$ cells $/ \mathrm{ml}$ were infected at a multiplicity of infection of $1-3$. After $3 \mathrm{~d}$, the culture medium was collected after centrifugation at $5000 \mathrm{~g}$ for $15 \mathrm{~min}$.

The culture medium was loaded onto a hydroxyapatite (Nacalai Tesque, Kyoto, Japan) column (bed volume: $10 \mathrm{ml}$ ) pre-equilibrated with $5 \mathrm{~mm}$ phosphate buffer ( $\mathrm{pH}$ 7.5). After extensive washing with the same buffer, laeverin/APQ was eluted from the column with $100 \mathrm{~mm}$ phosphate buffer $(\mathrm{pH}$ 7.5). The eluate was then loaded onto a $\mathrm{Ni}^{2+}$-chelating Sepharose (Pharmacia, Uppsala, Sweden) column (bed volume: $1 \mathrm{ml}$ ) pre-equilibrated with $10 \mathrm{~mm}$ phosphate buffer $(\mathrm{pH}$ 7.5) containing $0.1 \mathrm{M} \mathrm{NaCl}$. After extensive washing with $10 \mathrm{~mm}$ phosphate buffer ( $\mathrm{pH} 7.5$ ) containing $0.1 \mathrm{M} \mathrm{NaCl}$ and $5 \mathrm{~mm}$ imidazole, laeverin/APQ was eluted with $10 \mathrm{~mm}$ phosphate buffer ( $\mathrm{pH} 7.5$ ) containing $0.1 \mathrm{M} \mathrm{NaCl}$ and $150 \mathrm{~mm}$ imidazole. The laeverin/APQ-containing fractions were extensively dialyzed against $25 \mathrm{~mm}$ Tris-HCl buffer ( $\mathrm{pH} 7.5)$ containing $0.125 \mathrm{M} \mathrm{NaCl}$, concentrated with an ultrafiltration membrane and stored at $-20{ }^{\circ} \mathrm{C}$ prior to use.

Measurement of Aminopeptidase Activity of Laeverin/APQs Aminopeptidase activities of wild-type and mutant laeverin/APQs were routinely determined by endpoint assay with various fluorogenic aminoacyl-4-metheylcoumaryl-7-amides (aminoacyl-MCAs) as substrates. The reaction mixture containing $100 \mu \mathrm{M}$ aminoacyl-MCA and $1 \mu \mathrm{g} / \mathrm{ml}$ enzyme in $0.5 \mathrm{ml}$ of $25 \mathrm{~mm}$ Tris/ $\mathrm{HCl}$ buffer ( $\mathrm{pH} 7.5$ ) was incubated at $37^{\circ} \mathrm{C}$ for $15 \mathrm{~min}$. The reaction was terminated by adding $2.5 \mathrm{ml}$ of $0.1 \mathrm{M}$ sodium acetate buffer $(\mathrm{pH}$ 4.3) containing $0.1 \mathrm{M}$ sodium monochloroacetate. The amount of 7-amino-4-methylcoumarin released was measured by spectrofluorophotometry (F-2000; Hitachi) at an excitation wavelength of $360 \mathrm{~nm}$ and an emission wavelength of $460 \mathrm{~nm}$, and is given in arbitrary units.

To determine the kinetic parameters, the reaction mixture containing various concentrations of substrates and either wild-type or mutant laeverin/APQ in $500 \mu \mathrm{l}$ of $25 \mathrm{~mm}$ Tris/ $\mathrm{HCl}$ buffer $(\mathrm{pH} 7.5)$ was incubated at $37^{\circ} \mathrm{C}$ for $5 \mathrm{~min}$. The amount of 7-amino-4-methylcoumarin released was then measured. The kinetic parameters $\left(K_{\mathrm{m}}\right.$ and $\left.V_{\max }\right)$ were calculated using Lineweaver-Burk plots.

Materials Asp-, benzyl-Cys-, and Glu-MCAs were purchased from BACHEM AG (Bubendorf, Switzerland). Ala-, Arg-, Leu-, Lys- Met, and Phe-MCAs were obtained from the Peptide Institute (Osaka, Japan). Bestatin was purchased from Sigma.

\section{RESULTS AND DISCUSSION}

Molecular Modeling of the Catalytic Pocket of Laeverin/APQ In the previous work, we showed that Gln-181 of ERAP1 was critical for the enzymatic activity and substrate specificity of ERAP1. ${ }^{17)}$ Alignment of ERAP1 and laeverin/APQ indicates that Gln-238 of laeverin/APQ occupies the corresponding site of Gln-181 of ERAP1. Using TIFF3 as a template, we modeled the structure of the catalytic pocket of human laeverin/APQ (Fig. 1). As in the case of ERAP1, Gln-238 of laeverin/APQ is expected to be located near the catalytic $\operatorname{HEXXH}(\mathrm{X})_{18} \mathrm{E}$ motif and at a possible S1 site of the enzyme, suggesting the importance of this residue for enzymatic activity. To elucidate the role of Gln238 of human laeverin/APQ in its enzymatic properties further, this residue was substituted with Ala to generate Q238A laeverin/APQ, and its enzymatic property was compared with wild-type enzyme. We chose an Ala mutant to check the significance of Gln-238 residue, because the substitution of Gln-181 residue with Ala caused almost complete loss of the enzymatic activity of ERAP1, thus indicating the essential role of this residue in the enzymatic activity. ${ }^{17)}$

Characterization of the Hydrolytic Activities of WildType and Q238A Laeverin/APQs Figure 2 shows the enzymatic activities of wild-type and Q238A laeverin/APQs toward various substrates. As shown previously, the wildtype enzyme cleaved Leu-MCA most efficiently, followed by Met-, Arg- and Lys-MCA. Hydrolysis of Ala-, Phe-, and benzyl-Cys-MCA was also observed. On the other hand, in sharp contrast to Q181A ERAP1, which showed little hydrolytic activity toward substrates tested, Q238A laeverin/APQ retained substantial activity toward basic amino acid substrates, Arg and Lys-MCA. Little activity was detected toward other substrates tested. These results indicate that while wild-type enzyme shows broad substrate specificity, enzymatic activity of the mutant enzyme is rather restricted to basic amino acids.

Table 1 shows the kinetic parameters of wild-type and Q238A laeverin/APQs using Leu-MCA and Arg-MCA as substrates. The calculated $k_{\mathrm{cat}} / K_{\mathrm{m}}$ value from a LineweaverBurk plot of wild-type enzyme for the most efficient substrate Leu-MCA was $451 \pm 16 \mu \mathrm{M}^{-1} \mathrm{~s}^{-1}$. The catalytic efficiency $\left(k_{\mathrm{cat}} / K_{\mathrm{m}}\right)$ of the wild-type enzyme for Arg was $205 \pm 33 \mu \mathrm{m}^{-1} \mathrm{~s}^{-1}$, about 2.3-fold less than for Leu-MCA. On the other hand, when comparing the hydrolytic activity of mutant enzyme with that of the wild-type toward Arg-MCA, mutant enzyme showed comparable efficiency (232 \pm 49 $\mu \mathrm{M}^{-1} \mathrm{~s}^{-1}$ ). Both $K_{\mathrm{m}}$ and $k_{\text {cat }}$ values of mutant laeverin/APQ were about 2-fold less than those of wild-type enzyme, suggesting that replacement of Gln-238 with Ala slightly changed the substrate recognition mechanism toward basic amino acids. It showed little activity toward Leu-MCA with $k_{\text {cat }} / K_{\mathrm{m}}$ value of $2.79 \pm 0.12 \mu \mathrm{M}^{-1} \mathrm{~s}^{-1}$, because of a marked decrease in the $k_{\text {cat }}$ value. These results indicate that mutant enzyme retained substantial enzymatic activity toward ArgMCA and its substrate specificity was different from that of the wild-type enzyme and showed rather a restricted pattern. Taken together, we concluded that the substitution of Gln238 with Ala caused a change of substrate specificity rather than total decrease of the enzymatic activity toward substrates, which is quite different from the case of ERAP $1 .{ }^{17)}$ 


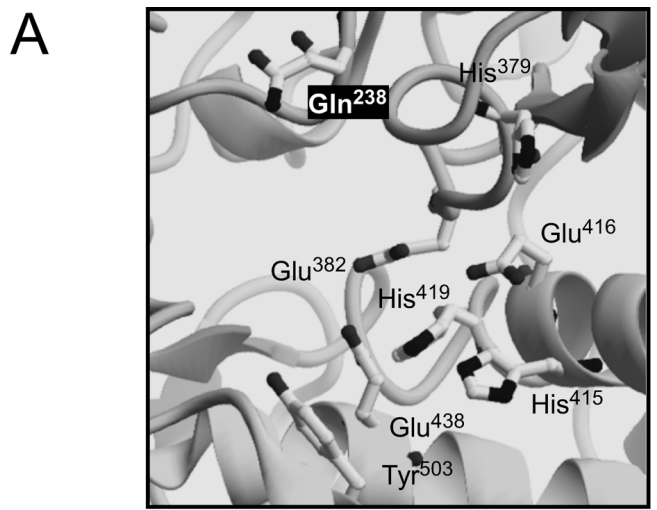

\section{B}

$\begin{array}{ll}\text { Laeverin/APQ } & 211 \\ \text { APN } & 185 \\ \text { APA } & 195 \\ \text { A-LAP/ERAP1 } & 154 \\ \text { P-LAP/IRAP } & 266 \\ \text { L-RAP/ERAP2 } & 171 \\ \text { PSA } & 151 \\ \text { TRHDE } & 240 \\ \text { APB } & 145 \\ \text { LTA4H } & 108 \\ \text { APO } & 281\end{array}$

238

LVKEDLREGLFLNVYTDQGERRALLASQLEPTFARYVFPCFDEPALKATFNITMIHHPSY 270 ELADDLAGFYRSEYME-GNVRKVVATTQMQAADARKSFPCFDEPAMKAEFNITLIHPKDL 243 WLNGSLVGFYRTTYTE-NGRVKSIVATDHEPTDARKSFPCFDEPNKKATYTISITHPKEY 253 NLSETFHGFYKSTYRTKEGELRILASTQFEPTAARMAFPCFDEPAFKASFSIKIRREPRH 213 NISSSYYGFYGFSYTDESNEKKYFAATQFEPLAARSAFPCFDEPAFKATFIIKIIRDEQY 325 KLGDGFEGFYKSTYRTLGGETRILAVTDFEPTQARMAFPCFDEPLFKANFSIKIRRESRH 230 ELNDKMKGFYRSKYTTPSGEVRYAAVTQFEATDARRAFPCWDEPAIKATFDISLVVPKDR 210 LIENELLGFFRSSYVL-HGERRFLGVTQFSPTHARKAFPCFDEPIYKATFKISIKHQATY 298 GEGP---GVCWLAPEQTAGKKKPFVYTQGQAVLNRAFFPCFDTPAVKYKYSALIEVPDGF 201 ETSPKSSALQWLTPEQTSGKEHPYLFSQCQAIHCRAILPCQDTPSVKLTYTAEVSVPKEL 167 LFPCQEPPVAMSTWQATVRAAASFVVLMSGENSAKPTQLWEECSSWYYYVTMPMPASTFT 340

Fig. 1. Molecular Modeling of the Catalytic Pocket of Laeverin/APQ

(A) Modeling of the catalytic site of human laeverin/APQ using Thermoplasma acidophilum TIFF3 as a template. His-379 and Glu-382 in the exopeptidase HAMEN motif and His-415, Glu-416, His-419 and Glu-438 in $\operatorname{HEXXH}(\mathrm{X})_{18} \mathrm{E}$ motif and Tyr-503 are shown as residues in the catalytic pocket of laeverin/APQ. (B) Alignment of the laeverin/APQ amino acid sequence with the sequences of the M1 family of aminopeptidases. Residues corresponding to Gln-238 of laeverin/APQ are shown as a white Q on a black background. Residues conserved among the enzymes are shaded.
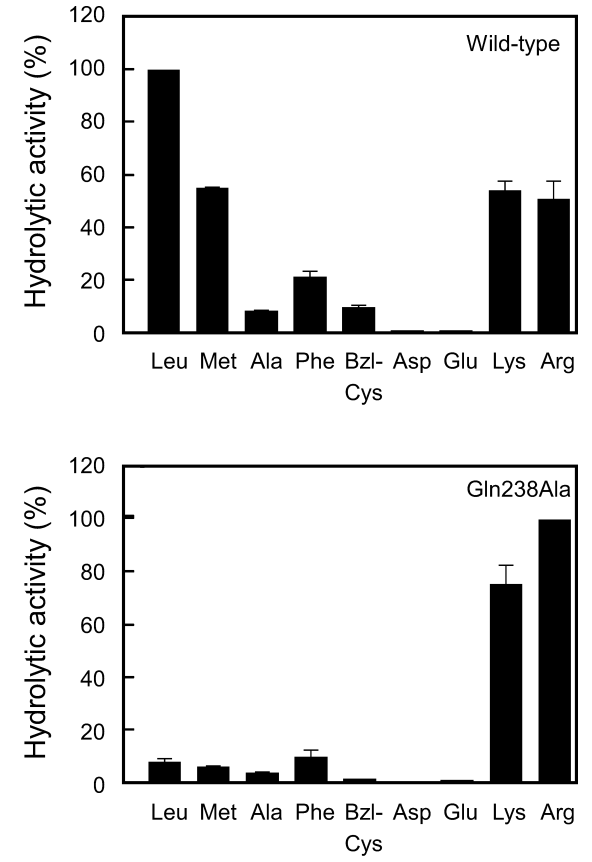

Fig. 2. Enzymatic Activities of Wild-Type and Q238A Laeverin/APQs toward Various Substrates

Purified enzymes $(1 \mu \mathrm{g} / \mathrm{ml})$ were incubated with various amino acid MCA substrates $(100 \mu \mathrm{M})$ at $37^{\circ} \mathrm{C}$ for $15 \mathrm{~min}$. Relative activities of the enzymes are shown, taking the hydrolytic activity of wild-type enzyme toward Leu-MCA (A) and Q238A mutant enzyme toward Arg-MCA (B) as 100\%. Each bar shows the mean \pm S.D. $(n=3)$.
Table 1. Kinetic Parameters of Wild-Type and Q238A Laeverin/APQs toward Leu-MCA and Arg-MCA

\begin{tabular}{ccccc}
\hline \hline Substrate & & $K_{\mathrm{m}}(\mu \mathrm{M})^{a)}$ & $k_{\text {cat }}\left(\mathrm{s}^{-1}\right)^{a)}$ & $\begin{array}{c}k_{\mathrm{cat}} / K_{\mathrm{m}} \times 10^{3} \\
\left(\mu \mathrm{M}^{-1} \cdot \mathrm{s}^{-1}\right)^{a)}\end{array}$ \\
\hline Leu-MCA & Wild-type & $119 \pm 21.9$ & $53.5 \pm 8.06$ & $451 \pm 15.7$ \\
& Q238A & $83.9 \pm 6.70$ & $0.23 \pm 0.01$ & $2.79 \pm 0.12$ \\
Arg-MCA & Wild-type & $27.9 \pm 7.82$ & $5.56 \pm 0.81$ & $205 \pm 32.9$ \\
& Q238A & $11.5 \pm 4.33$ & $2.53 \pm 0.52$ & $232 \pm 49.4$ \\
\hline
\end{tabular}

a) Values are mean \pm S.D. $(n=3)$.

Effect of Bestatin on the Enzymatic Activities of WildType and Q238A Laeverin/APQs Because laeverin/APQ is unique in that bestatin inhibits its enzymatic activity more efficiently than amastatin, ${ }^{15)}$ we next compared the effect of bestatin on the enzymatic activity of the wild-type and Q238A laeverin/APQs. As shown in Fig. 3, bestatin inhibited the enzymatic activity of the wild-type enzyme toward LeuMCA comparably with that of toward Arg-MCA. On the other hand, it inhibited the mutant enzyme about 50-fold less efficiently than the wild-type enzyme when measured using Arg-MCA as a substrate. Considering that bestatin $(N$ [(2S,3R)-3-amino-2-hydoroxy-4-phenylbutyryl]-L-leucine hydorochloride) is a small peptide having an aromatic amino acid-like residue at its $\mathrm{N}$-terminus, consistent with the notion that wild-type laeverin/APQ binds to aromatic amino acids with higher affinity than the Q238A mutant enzyme. 


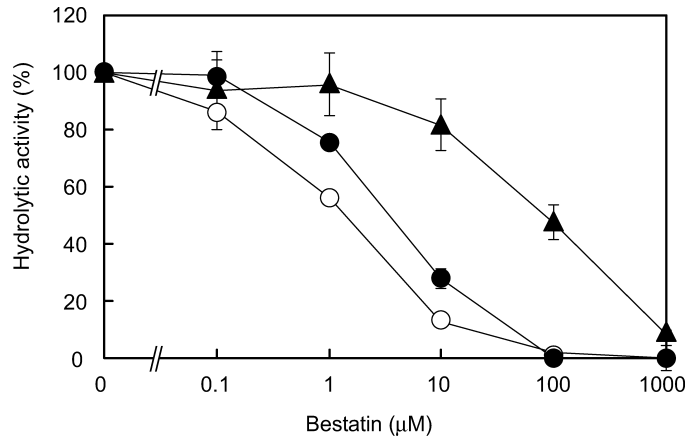

Fig. 3. Effect of Bestatin on the Enzymatic Activity of Wild-Type and Q238A Laeverin/APQs

Purified wild-type and Q238A laeverin/APQs were incubated with various concentrations of bestatin on ice for $5 \mathrm{~min}$. Aminopeptidase activities were then measured using Leu-MCA $(100 \mu \mathrm{M})(\bigcirc)$ or Arg-MCA $(100 \mu \mathrm{M})(\bullet)$ as the wild-type and ArgMCA $(100 \mu \mathrm{M})(\boldsymbol{\Lambda})$ as Q238A laeverin/APQ.

In this study, we identified Gln-238 as a residue crucial in the substrate specificity of human laeverin/APQ. Replacement of this residue with Ala caused the enzyme preference for basic amino acids and rather restricted substrate specificity. In our previous work, we reported that replacement of Gln-181 of human ERAP1 with Asp increased the enzyme preference for basic amino acid. ${ }^{17)}$ We speculated that replacement of Gln-181 with acidic amino acids might cause a local conformational change of the substrate pocket, resulting in rather restricted substrate specificity through electrostatic interaction between the residue and $\mathrm{N}$-terminal basic amino acid of the substrate. However, replacement of Gln238 of human laeverin/APQ with neutral amino acid Ala caused the enzyme preference for basic amino acids, suggesting that the direct electrostatic interaction between Ala238 and N-terminal basic amino acid of the substrates may have little role in the basic amino acid preference of the enzyme. Instead, we speculate at present that both Gln-238 of wild-type and Ala-238 of Q238A mutant laeverin/APQs maintain their catalytic pocket structures by interacting or interfering with another unidentified residues, and thus form unique S1 sites, as discussed elsewhere. ${ }^{17)}$ It is possible that unique S1 site structure of the mutant enzyme but not that of the wild-type may allow the electrostatic interaction between neighboring acidic amino acid and basic amino acid substrate, causing the basic amino acid preference.

The corresponding site of the M1 family of aminopeptidases is occupied either Gln or Asp (Fig. 1B). When the site is occupied by Asp, this site may interact with either $\mathrm{Ca}^{2+}$ $(\mathrm{APA})^{19)}$ or substrates (L-RAP/ERAP2) ${ }^{20)}$ through electro- static interaction and thus contribute to the unique properties of the enzymes. To elucidate the mode of the interaction between S1 site and substrates definitively, it is essential to determine the crystal structure of the enzymes.

In summary, through substitution of Gln-238 with Ala of human laeverin/APQ, we have shown that this residue is important for its substrate specificity; however, as direct electrostatic interaction is not likely to be involved in the interaction between N-terminal amino acid of the substrate and S1 site of the enzyme, it is conceivable that another acidic residue might participate in their interaction.

\section{REFERENCES}

1) Taylor A., FASEB J., 7, 290-298 (1993).

2) Hooper N. M., FEBS Lett., 354, 1-6 (1994).

3) Tsujimoto M., Hattori A., Biochim. Biophys. Acta, 1751, 9-18 (2005).

4) Sato Y., Biol. Pharm. Bull., 27, 772-776 (2004).

5) Constam D. B., Tobler A. R., Rensing-Ehl A., Kemler I., Hersh L. B., Fontana A., J. Biol. Chem., 270, 26931-26939 (1995).

6) Osada T., Watanabe G., Kondo S., Toyoda M., Sakaki Y., Takeuchi T., Mol. Endocrinol., 15, 960-971 (2001).

7) Albiston A. L., McDowall S. G., Matsacos D., Sim P., Clune E., Mustafa T., Lee J., Mendelsohn F. A. O., Simpson R. J., Connolly L. M., Chai S. Y., J. Biol. Chem., 276, 48623-48626 (2001).

8) Goto Y., Hattori A., Ishii Y., Tsujimoto M., FEBS Lett., 580, 1833 1838 (2006).

9) Wright J. W., Mizutani S., Murray C. E., Amir H. Z., Harding J. W., J. Hypertens., 8, 969-974 (1990).

10) Reaux A., Fournie-Zaluski M. C., David C., Zini S., Roques B., Corvol P., Llorens-Cortes C., Proc. Natl. Acad. Sci. U.S.A., 96, 1341513420 (1999).

11) Serwold T., Gonzalez F., Kim J., Jacob R., Shastri N., Nature (London), 419, 480-483 (2002).

12) Saric T., Chang S.-C., Hattori A., York I. A., Markant S., Rock K. L., Tsujimoto M., Goldberg A. L., Nat. Immunol., 3, 1169-1176 (2002).

13) Fujiwara H., Higuchi T., Yamada S., Hirano T., Sato Y., Nishioka Y., Yoshioka S., Tatsumi K., Ueda M., Maeda M., Fujii S., Biochem. Biophys. Res. Commun., 313, 962-968 (2004).

14) Puente X. S., Sanchez L. M., Overall C. M., Lopez-Otin C., Nat. Rev. Genet., 4, 544-558 (2003).

15) Maruyama M., Hattori A., Goto Y., Ueda M., Maeda M., Fujiwara H., Tsujimoto M., J. Biol. Chem., 82, 20088-20096 (2007).

16) Maruyama M., Arisaka N., Goto Y., Ohsawa Y., Inoue H., Fujiwara H., Hattori A., Tsujimoto M., J. Biol. Chem., 284, 34692-34702 (2009).

17) Goto Y., Tanji H., Hattori A., Tsujimoto M., Biochem. J., 416, 109116 (2009).

18) Kyrieleis O. J. P., Goettig P., Kiefersauer R., Huber R., Brandstetter H., J. Mol. Biol., 349, 787-800 (2005).

19) Goto Y., Hattori A., Mizutani S., Tsujimoto M., J. Biol. Chem., 282, 37074-37081 (2007).

20) Tanioka T., Hattori A., Masuda S., Nomura Y., Nakayama H., Mizutani S., Tsujimoto M., J. Biol. Chem., 278, 32275-32283 (2003). 